\title{
BMJ Open Phantom motor execution as a treatment for phantom limb pain: protocol of an international, double-blind, randomised controlled clinical trial
}

\author{
Eva Lendaro, ${ }^{1}$ Liselotte Hermansson, ${ }^{2,3}$ Helena Burger, ${ }^{4,5}$ Corry K Van der Sluis, ${ }^{6}$ \\ Brian E McGuire, ${ }^{7}$ Monika Pilch, ${ }^{7}$ Lina Bunketorp-Käll, ${ }^{8}$ Katarzyna Kulbacka-Ortiz, ${ }^{8}$ \\ Ingrid Rignér, ${ }^{9}$ Anita Stockselius, ${ }^{10}$ Lena Gudmundson, ${ }^{10}$ Cathrine Widehammar, ${ }^{3}$ \\ Wendy Hill, ${ }^{11}$ Sybille Geers, ${ }^{12}$ Max Ortiz-Catalan ${ }^{1,13}$
}

To cite: Lendaro $\mathrm{E}$, Hermansson L, Burger $\mathrm{H}$, et al. Phantom motor execution as a treatment for phantom limb pain: protocol of an international, doubleblind, randomised controlled clinical trial. BMJ Open 2018;8:e021039. doi:10.1136/ bmjopen-2017-021039

- Prepublication history and additional material for this paper are available online. To view these files, please visit the journal online (http://dx.doi. org/10.1136/bmjopen-2017021039).

Received 19 December 2017 Revised 3 April 2018 Accepted 20 April 2018

Check for updates

For numbered affiliations see end of article.

Correspondence to Dr Max Ortiz-Catalan; maxo@chalmers.se

\section{ABSTRACT}

Introduction Phantom limb pain (PLP) is a chronic condition that can greatly diminish quality of life. Control over the phantom limb and exercise of such control have been hypothesised to reverse maladaptive brain changes correlated to PLP. Preliminary investigations have shown that decoding motor volition using myoelectric pattern recognition, while providing real-time feedback via virtual and augmented reality (VR-AR), facilitates phantom motor execution (PME) and reduces PLP. Here we present the study protocol for an international (seven countries), multicentre (nine clinics), double-blind, randomised controlled clinical trial to assess the effectiveness of PME in alleviating PLP.

Methods and analysis Sixty-seven subjects suffering from PLP in upper or lower limbs are randomly assigned to PME or phantom motor imagery (PMI) interventions. Subjects allocated to either treatment receive 15 interventions and are exposed to the same VR-AR environments using the same device. The only difference between interventions is whether phantom movements are actually performed (PME) or just imagined (PMI). Complete evaluations are conducted at baseline and at intervention completion, as well as 1, 3 and 6 months later using an intention-to-treat (ITT) approach. Changes in PLP measured using the Pain Rating Index between the first and last session are the primary measure of efficacy. Secondary outcomes include: frequency, duration, quality of pain, intrusion of pain in activities of daily living and sleep, disability associated to pain, pain self-efficacy, frequency of depressed mood, presence of catastrophising thinking, health-related quality of life and clinically significant change as patient's own impression. Followup interviews are conducted up to 6 months after the treatment.

Ethics and dissemination The study is performed in agreement with the Declaration of Helsinki and under approval by the governing ethical committees of each participating clinic. The results will be published according to the Consolidated Standards of Reporting Trials guidelines in a peer-reviewed journal. Trial registration number NCT03112928; Pre-results.

\section{Strengths and limitations of this study}

- This study involves a suitable number of participants $(>60)$ to provide the power necessary for meaningful conclusions.

- This study is double-blinded, randomised controlled clinical trial, conducted in geographically different locations and involves subjects with both upper and lower limb amputations, thus enhancing generalisability.

- The choice of the comparator allows controlling in a stringent manner for the effect of the key factor hypothesised as the cause of pain reduction, namely, the execution of phantom limb movements.

- Treatment is limited to 15 sessions, which might not be enough to alleviate pain in all participants.

- The nature of the experimental treatment (phantom motor execution) does not allow inclusion of individuals from which myoelectric signals cannot be recorded from the muscles in their residual limbs.

\section{INTRODUCTION}

Phantom limb pain (PLP) is a chronic condition commonly suffered by amputees. ${ }^{1} 2$ Although more than 60 different treatments to alleviate PLP have been described in the literature, ${ }^{3}$ controlled clinical trials on such treatments are scarce and tend to be of poor quality. ${ }^{4}$ The clinical investigation presented in this protocol aims to evaluate the efficacy of phantom motor execution (PME) in reducing PLP in an international, multicentre, double-blind, randomised controlled clinical (RCT) trial. PME is accomplished by using a system (Neuromotus, Integrum AB, Sweden) that employs myoelectric pattern recognition to predict motor volition (movements of the phantom limb) while providing real-time feedback to the patient in virtual and augmented reality (VR/AR) environments. 
This technology allows the application of serious gaming in the therapy. PME is a non-invasive, non-pharmacological and engaging treatment with no identified side effects at present. ${ }^{56}$

The effectiveness of PME was initially explored in a single upper limb amputee, with satisfactory results reported. ${ }^{5}$ Prior to the pilot study, the patient had shown resistance to a variety of treatments for 48 years (including mirror therapy). After PME, the sustained level of pain reported by the patient was gradually reduced to painfree periods. He and his family also reported less intrusion of PLP in sleep and activities of daily living (ADLs). Finally, the patient also acquired the ability to freely move his phantom arm and hand, consistent with a recent study by Raffin and colleagues ${ }^{7}$ where they found that reduced capability of phantom movement was correlated with more severe PLP.

In the light of the findings in the case study, a non-randomised clinical investigation on PME was conducted in subjects with chronic intractable upper limb PLP. ${ }^{6}$ Fourteen patients, for whom conventional PLP treatments failed and who suffered from PLP for an average of 10 years, received 12 treatment sessions of PME, each of 1.5-hour duration. At the end of the treatment period, patients showed statistically and clinically significant improvements (approximately 50\% reduction of PLP). Intrusion of PLP during sleep and ADL was also reduced by a similar degree. These improvements were still present up to 6 months' post-treatment. ${ }^{6}$ More recently, PME was also demonstrated to be a viable treatment for PLP in lower limb amputations. ${ }^{8}$

Strong evidence shows that PLP is related to neuroplastic changes in the primary somatosensory cortex, suggesting that central maladaptive plasticity is responsible for its maintenance. Neuroplasticity-based approaches for the relief of PLP, such as motor imagery and mirror therapy, ultimately aim to regain brain circuitry from pain processing. Nonetheless, these approaches have been shown to be limited in their effectiveness.

Although the practice of motor imagery has been shown to normalise previously altered cortical maps and reduce PLP, ${ }^{9}$ evidence from randomised clinical studies has also suggested that it can increase pain. ${ }^{10}$ These seemingly contradictory findings suggest that motor imagery should not be used alone but combined with other interventions, such as graded motor imagery ${ }^{11}$ or mirror therapy. ${ }^{12}$

Mirror therapy has demonstrated higher effectiveness than motor imagery in reducing pain ${ }^{10}$; however, it still cannot ensure that the patient performs movements with the phantom limb. For instance, it is enough for the patient to move their healthy arm to produce movement in the reflected limb. Whether a patient is actually engaging in execution of phantom limb movements is unknown. PME overcomes some of the methodological limitations of previous treatments by ensuring that central and peripheral mechanisms in motor control are activated during therapy.
Table 1 List of the investigational sites, divided by countries taking part to the international, multicentre randomised clinical trial

\begin{tabular}{|c|c|}
\hline Country & Investigational site \\
\hline \multirow[t]{3}{*}{ Sweden } & Sahlgrenska University Hospital, Gothenburg \\
\hline & Örebro University Hospital, Örebro \\
\hline & $\begin{array}{l}\text { Rehabcenter Sfären, Bräcke Diakoni, } \\
\text { Stockholm }\end{array}$ \\
\hline Slovenia & University Rehabilitation Institute, Ljubljana \\
\hline Belgium & $\begin{array}{l}\text { Fysische Geneeskunde en Revalidatie } \\
\text { University Hospital Gent, Gent }\end{array}$ \\
\hline $\begin{array}{l}\text { The } \\
\text { Netherlands }\end{array}$ & $\begin{array}{l}\text { Department of Rehabilitation Medicine, } \\
\text { University Medical Centre Groningen, } \\
\text { Groningen }\end{array}$ \\
\hline Canada & $\begin{array}{l}\text { Institute of Biomedical Engineering, University } \\
\text { of New Brunswick, New Brunswick }\end{array}$ \\
\hline Ireland & $\begin{array}{l}\text { Centre for Pain Research, National University } \\
\text { of Ireland, Galway }\end{array}$ \\
\hline Germany & $\begin{array}{l}\text { Department of Psychosomatic Medicine and } \\
\text { Psychotherapy, LWL University Hospital, } \\
\text { Ruhr-University Bochum, Bochum }\end{array}$ \\
\hline
\end{tabular}

\section{STUDY OBJECTIVE}

This paper presents the study protocol for a RCT in which upper and lower limb amputees are treated. The investigation primarily aims at assessing the efficacy of PME aided by myoelectric pattern recognition, VR/AR and serious gaming to reduce PLP. In order to isolate the contribution of PME in alleviating PLP over potential placebo effects, phantom motor imagery (PMI) is used in this study as an active control treatment.

The working hypothesis of PME is that execution of phantom limb movements would exploit competitive neuroplasticity and provide a more integral normalisation of cortical, subcortical and spinal circuits compared with interventions that do not enable integration of sensory and motor information. Therefore, in this superiority trial, we hypothesise that the participants receiving the experimental treatment (PME) to obtain a larger reduction in PLP levels than those randomised to the control treatment.

\section{TRIAL DESIGN}

This clinical study is an international, multicentre, doubleblind, randomised controlled trial. The study takes place in seven counties and involves nine clinics, which are listed in table 1. Participants are randomly assigned to receive either the experimental or the control treatment in a 2:1 allocation ratio. The choice of the allocation ration was made in order to collect more data on the intervention of interest. Each patient is followed up for a period of 6 months, at the end of which they are given the choice to undergo the alternative treatment. The total duration of the study is expected to be approximately 3 years. 


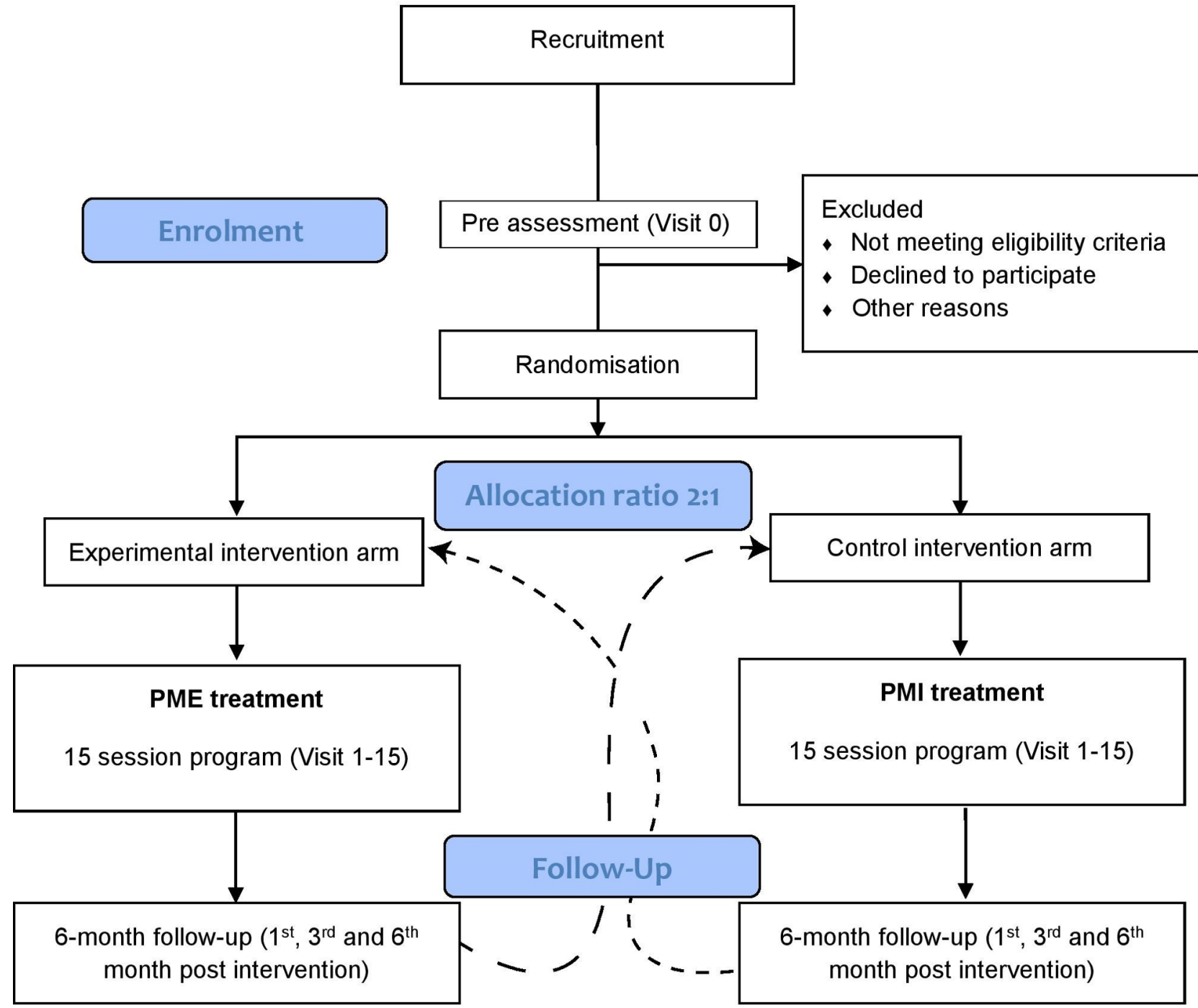

Figure 1 Flow diagram for the randomised controlled clinical trial. At least 67 patients are recruited and randomly allocated to either phantom motor execution (PME) or phantom motor imagery (PMI) interventions in allocation ratio 2:1. Following the completion of the treatment protocol and wash-out period of 6 months, it is possible for the patient to cross over to the parallel interventional arm, according to their will.

\section{METHODS: PARTICIPANTS, INTERVENTIONS, AND OUTCOMES}

A procedural overview of the trial is provided by the flow diagram of figure 1 . Recruitment of the participants is conducted via advertisements at local investigation clinics, on social media and in local newspapers. People who are interested in taking part in the trial are invited to contact the principal investigator of the site, or a person appointed by the principal investigator, via phone or email.

\section{Eligibility criteria}

Interested people are invited to a preassessment visit (visit $0)$. On this occasion, the therapist (clinical investigator) explains the study in detail and answers all the questions that might arise. Afterwards, the participants are asked to provide written informed consent (see supplementary appendix A). If consent is granted, eligibility to the study is assessed according to the criteria presented below:
- The participants must be older than 18 years with chronic PLP.

- Participants must have chronic PLP-at least 6 months should have passed since amputation. Participants with acute PLP are non-eligible.

- In case of pharmacological treatments, the dosage must have been stable for the previous month.

- Any previous PLP treatments must have terminated at least 3 months prior to entering the study.

- Any pain reduction potentially attributable to previous PLP treatments must have occurred at least 3 months prior to entering the study.

- Voluntary control over at least a portion of biceps and triceps muscles in case of upper limb amputation or quadriceps and hamstrings in case of lower limb amputation.

- Stable prosthetic situation (ie, satisfaction with the fitting of the prosthesis) or being a non-user. 
- The subject should not have a cognitive impairment that prevents them from following instructions.

- No abundant soft tissue on the stump that prevents sufficient myoelectric signals from being recorded.

- No presence of pain >2 on Numerical Rating Scale (NRS) on contact with the skin or muscle contraction in the stump.

- The PLP must not be aggravated (NRS $>4$ ) by the execution or imagination of phantom movements.

- No condition associated with risk of poor protocol compliance.

- No injury, disease or addiction that would render the individual unsuitable for the trial.

- Pain Rating Index (PRI) $>0$ as assessed in the Questionnaire for Phantom Limb Pain (Q-PLP) at visit 0.

\section{Concomitant medications}

Any cointervention aiming to reduce PLP is prohibited during the trial. However, in the design of the trial, it is acknowledged that there is a large possibility for patients with PLP to be high consumers of analgesic medicines. Therefore, the use of concomitant medications is allowed provided that at the time of inclusion, the patient has stable consumption for at least 1 month before entering the study and any pain reduction potentially attributable to the drug occurred at least 3 months before entering the study. Intake of pain medication in patients who show considerable improvement can be gradually reduced at the discretion of the responsible physician, given that the patient is followed up regularly. Medication intake is thus monitored as an outcome variable called 'need of concomitant medication', which is used to describe and compare the amount of comedication in the treatment groups.

\section{Interventions}

All of the therapists at the clinics are introduced to the technology with at least one practical demonstration by the first (EL) and/or last author (MO-C). The therapists conduct the interventions independently, and periodically the first author monitors the correct execution of the protocol. Participants in both intervention groups receive 15 treatment sessions of 2 hours' duration, including system setup and a blinded outcome assessment. The frequency of the sessions is chosen by the participant and can be once, twice (advised frequency) or five times per week, yielding a total patient duration that ranges between 28 and 40 weeks. Both treatment groups use the same device and setup, which are sketched in figure 2. The only difference between the two groups is the type of interaction with the virtual environments (active: motor execution; or passive: motor imagery). Allocated interventions for a given trial participant cannot be modified. Dates of the treatment sessions are recorded.

\section{Experimental treatment}

In the PME intervention, motor volition is decoded by interpreting the signals from the stump muscles via

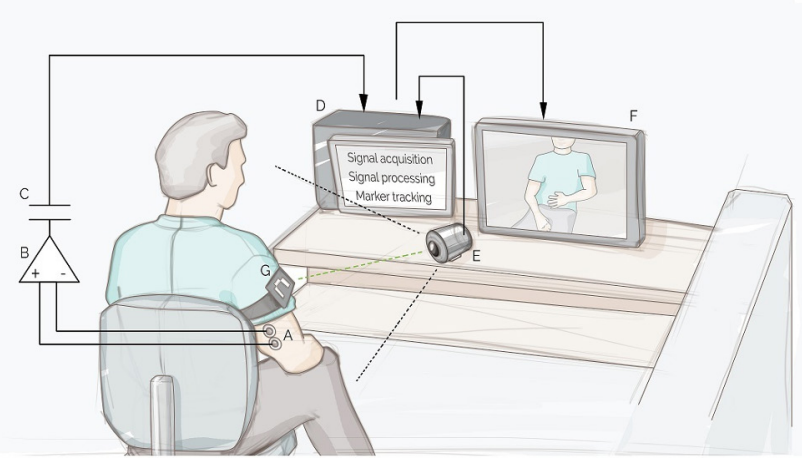

Figure 2 Schematic illustration of the clinical investigation device with all its components. Myoelectric signals are acquired though surface electrodes $(A)$ by a myoelectric amplifier (B), electrically isolated (C). The signals are then processed by the software installed on the computer (D). The camera (E) films the participant and the recorded image is displayed on the monitor $(F)$ with a virtual limb superimposed where the marker $(G)$ is detected. Figure courtesy of Jason Millenaar.

myoelectric pattern recognition. ${ }^{13} 14$ The decoded movement is visualised in the virtual environments (ie, virtual limb or serious gaming). The end result is that the user, by training with the system, can achieve control over the virtual environments by performing phantom limb movements associated with kinetic sensations analogous to the ones pertaining to the limb prior to amputation.

A treatment session consists of the following steps:

1. Placement of the electrodes and fiducial marker.

2. Treatment cycles

a. Recording session.

b. Practice of PME with VR/AR.

c. Serious gaming using phantom movements.

d. Practice of PME by matching random target postures of a virtual arm in VR (TAC Test ${ }^{15}$ ).

3. Pain evaluation (Q-PLP; see Outcomes section).

Different treatment cycles (step 2) are repeated during a treatment session in order to execute various phantom limb movements or combinations of movements. The level of difficulty gradually increases during the treatment phase from 1 to 5 by adding $\mathrm{df}$ to be trained within the same treatment cycle. In this context, a df is any pair of movements performing opposite actions such as opening and closing of the hand or extension and flexion of the knee.

Clinicians are instructed to advance the level of difficulty once the previous level is accomplished successfully and revert to the previous level if the patient shows considerable difficulty accomplishing the tasks. More details on the acquisition of myoelectric signals, prediction of motor volition, the various parts of the treatment session and the different levels of difficulty are presented in online supplementary appendix B.

\section{Control treatment}

In the control treatment (PMI), patients are not allowed to produce/execute phantom movements, but must imagine performing such movements while observing them 
executed autonomously by the VR/AR environments. The device is identical to the one used in the experimental treatment, but here the myoelectric signals are used to monitor that the patient does not produce muscular contractions, rather than decoding motor volition.

The control treatment session is conducted using the same stepwise procedure as the experimental group with the addition of a calibration step at the beginning of the treatment cycle. Calibration is necessary to set the threshold for myoelectric signals above, which the system alerts the user that a muscular contraction is performed. As in PME, the treatment cycle is repeated for different imaginary phantom limb movements or a set of imaginary movements following the same levels of difficulty. In the game format, the participants control the game using the keyboard with an able limb. Bilateral upper limb amputees use a joystick with any able limb. Details on the methods are presented in online supplementary appendix B.

\section{Withdrawal or termination of individual participants}

Participants are free to withdraw from participation in the study at any time on request. An investigator may terminate participation in the study if:

- Any clinical adverse event, clinical abnormality or other medical condition or situation occurs such that continued participation in the study would not be in the best interest of the participant.

- The participant no longer meets the eligibility criteria because of a condition newly developed or not previously recognised.

The main analysis will be conducted using the intention-to-treat (ITT) methodology. Missing data due to withdrawal or termination will be imputed using the 'last observation carried forward' method. From previous studies, the dropout rate is estimated at approximately $10 \%$, and this was taken into account for the calculation of the sample size.

\section{Outcomes}

Outcomes will be evaluated at every treatment session and three follow-up assessments at 1, 3 and 6 months' post-treatment. The outcomes are measured by the evaluators following the participant treatment schedule presented in table 2 .

\section{Primary outcome measure}

The primary outcome of the study is the change in PLP intensity measured by the difference in PRI between baseline (visit 0 ) and at the post-treatment assessment (visit 15). The PRI is computed as the sum of the scores for all descriptors of the Short Form of the McGill Pain Questionnaire (SF-MPQ). ${ }^{16}$ Within this study, the SF-MPQ is included in one more extensive survey named Questionnaire for Phantom Limb Pain, which is described below in the secondary outcome measures.

\section{Secondary outcome measures}

Secondary outcomes consider different aspects related to PLP such as pain frequency, pain duration, quality
Table 2 Summary of the different items (intervention, forms and questionnaires) to be completed at each evaluation appointment

\begin{tabular}{|c|c|}
\hline Session & Summary of content \\
\hline Visit 0 & $\begin{array}{l}\text { Patient information }(T) \text {. } \\
\text { Study consent }(T) \text {. } \\
\text { Preassessment }(T) \text {. } \\
\text { Background information }(T) . \\
\text { Q-PLP }(T) . \\
\text { PDI }(T) . \\
\text { EQ5D-5L }(T) . \\
\text { PSEQ-2 }(T) . \\
\text { PCS-SF }(T) . \\
\text { PHQ-2 }(T) . \\
\text { PXPECT-SF }(T) .\end{array}$ \\
\hline \multicolumn{2}{|l|}{ Randomisation } \\
\hline Visit 1 & $\begin{array}{l}\text { Treatment session }(T) . \\
\text { Q-PLP }(E) . \\
\text { OAT }(E) . \\
\text { EXPECT-SF }(E) . \\
\text { HCCQ-SF }(E) .\end{array}$ \\
\hline Visits 2-14 & $\begin{array}{l}\text { Treatment session }(\mathrm{T}) \text {. } \\
\text { Q-PLP }(\mathrm{E}) \text {. }\end{array}$ \\
\hline Visit 15 & $\begin{array}{l}\text { Treatment session (T). } \\
\text { Q-PLP (E). } \\
\text { PDI (E). } \\
\text { EQ5D-5L (E). } \\
\text { PSEQ-2 (E). } \\
\text { PCS-SF (E). } \\
\text { PHQ-2 (E). } \\
\text { P } \\
\text { PGIC (E). } \\
\text { HCCQ-SF (E). }\end{array}$ \\
\hline $\begin{array}{l}\text { 1-month follow-up } \\
\text { 3-month follow-up } \\
\text { 6-month follow-up }\end{array}$ & $\begin{array}{l}\text { Q-PLP (E). } \\
\text { PDI (E). } \\
\text { EQ5D-5L (E). } \\
\text { PSEQ-2 (E). } \\
\text { PCS-SF (E). } \\
\text { PHQ-2 (E). }\end{array}$ \\
\hline
\end{tabular}

The letter in brackets indicates whether the therapist (T) or the evaluator $(E)$ is responsible of conducting a particular item is EQ-5D-5L, EuroQol-5D-5L; EXPECT-SF, Expectations for Complementary and Alternative Medicine Treatments Short Form; HCCQ, Health Care Climate Questionnaire; OAT, Opinion About Treatment; PCS-SF, Pain Catastrophizing Scale Short Form; PDI, Pain Disability Index; PGIC, Patients' Global Impression of Change; PHQ-2, two-item Patient Health Questionnaire; PSEQ-2, twoitem Pain Self-Efficacy Questionnaire; Q-PLP, Questionnaire for Phantom Limb Pain.

of pain, intrusion of pain in ADLs and sleep, disability associated with pain, pain self-efficacy, mood, presence of catastrophising thinking, health-related quality of life and the patient's own impression about the effect of treatment. The secondary outcome measures are:

\section{Pain Disability Index (PDI)}

PDI, a seven-item questionnaire designed to investigate the extent to which chronic pain interferes with a person's ability to engage in various life activities. ${ }^{17} \mathrm{An}$ 
overall PDI score is obtained by summing the numerical ratings of the questionnaire's single items.

\section{Questionnaire for Phantom Limb Pain}

The Q-PLP is a 16-item questionnaire based on a combination of the SF-MPQ ${ }^{16}$ and study-specific questions use in previous studies. ${ }^{568}$. The part containing the SF-MPQ is used for the calculation of the PRI (primary outcome measure).

The Q-PLP assesses intensity, quality, duration and frequency of PLP using the following metrics: the numeric rating scale (scale range $0-10$ ) to assess the intensity of pain at present; the weighted pain distribution (scale range $0-5$ ) to capture the time-varying nature of chronic pain by adding the contributions of weighted portions of time spent in six pain levels (present pain intensity scale ${ }^{18}$ ); and a study-specific descriptive scale of seven steps: 'never', 'once per month', 'once per week', 'few times per week', 'once per day', 'few times per day' and 'always' to measure the frequency of pain.

In addition, the Q-PLP is used to monitor the intensity of stump pain, phantom limb sensations, phantom motor ability, intrusion of PLP in ADLs and sleep, by one question each using a numeric rating scale. Changes in prosthetic hardware, medication, presence of telescoping (feeling that the phantom limb is gradually shortening over time) and location of pain are also monitored by the Q-PLP.

\section{EuroQol-5D-5L (EQ-5D-5L)}

The EQ-5D-5L is a standardised questionnaire used to investigate health-related quality of life, which is constituted by two components: health status and health evaluation. ${ }^{19}$ Health status is measured in terms of five dimensions (mobility, self-care, usual activities, pain/ discomfort and anxiety/depression) on a five-point scale (no problems, slight problems, moderate problems, severe problems and extreme problems). In the health evaluation part, the EQ Visual Analogue Scale records the respondent's health on a vertical VAS where the end points are labelled 'best imaginable health state' and 'worst imaginable health state'.

\section{Pain Self-Efficacy Questionnaire (PSEQ-2)}

The PSEQ-2 is a two-item questionnaire that measures pain self-efficacy, which is the belief held by people with chronic pain that they can carry out certain activities and enjoy life, despite experiencing pain. ${ }^{20}{ }^{21}$ The items of the questionnaire are rated on a numeric rating scale from 0 to 6 .

\section{Pain Catastrophizing Scale - 6 (PCS-6)}

The PCS-6 is a six-item questionnaire that investigates catastrophising thinking in a range from 0 to $4 .^{22} 23$ Pain catastrophising denotes a negative cognitive-affective response to pain and is associated to increased pain severity, disability and depressive symptoms and is associated with poor adjustment to chronic pain. ${ }^{24}$

\section{Patient Health Questionnaire-2 (PHQ-2)}

The PHQ-2 is a screening instrument consisting of two items assessing the presence of a depressed mood and a loss of interest or pleasure in routine activities. ${ }^{25}{ }^{26}$ The items of the questionnaire are rated on a numerical scale from 0 to 3 .

\section{Patients' Global Impression of Change (PGIC)}

The PGIC is a single question used to identify clinically significant change by rating the patient's belief about the efficacy of treatment on a seven-point scale, ranging from 'no change (or condition has got worse)' to 'a great deal better'. ${ }^{27}$

\section{Additional measurements}

Participants are asked to supply details regarding background information such as age, gender, height, weight, type and use of the prosthesis, level of embodiment of the prosthesis, onset of PLP, details about previous and ongoing intervention for PLP and side, level and date of amputation. Additionally, we also survey: patients' expectancy of benefit using the Expectations for Complementary and Alternative Medicine Treatments (EXPECT-SF) $)^{28}$; patients' judgement about the credibility of the treatment using the Opinion About Treatment (OAT ${ }^{29}$ and patients' perception of therapists' supportive behaviour using the short form of the six-item Health Care Climate Questionnaire (HCCQ) ${ }^{30}$

\section{Sample size}

The calculation of the sample size was based on our primary hypothesis and informed by our previous clinical trial with no control group. ${ }^{6}$ In order to find a mean difference of 4 between the two randomised groups in the primary outcome measure (PRI), with power of $80 \%$ resulting from a two-sided Fisher's non-parametric permutation test at $5 \%$ significance level, is estimated that at least 60 participants are required. As a drop-out rate of $10 \%$ is expected, a total of 67 patients will be randomised.

\section{METHODS: ASSIGNMENT OF INTERVENTION Randomisation}

Participants are assigned to the experimental or control group according to the optimal allocation scheme of minimisation, aimed at reducing the imbalance between the number of patients allocated to each treatment group. The randomisation proportion is $2: 1$, with twice as many subjects assigned to the experimental treatment. The allocation ratio was chosen to collect more information on important variables regarding the intervention of interest. The allocation aims to minimise the imbalance of the following factors:

- Level of amputation (upper and lower).

- Baseline PLP based on the NRS (low 1-4, and high $5-10)$.

- Investigation site (nine centres). 
The minimisation process is conducted using the opensource desktop application MinimPy, ${ }^{31}$ operated by the monitor of the clinical trial. Every time a research team at a particular investigational site recruits a new participant, they assess the person's eligibility for the study (visit 0 ). Afterwards, if the participant is deemed eligible, the research team sends the minimisation factors relative to the enrolled participant to the monitor, who runs the randomisation and informs the research team of the allocation.

\section{Blinding}

This investigation has been designed in such a way that participants of the two treatment groups use the same device under the same circumstances.

Even though the patients are necessarily aware of the treatment they are receiving, they do not have an expectation of superiority of the experimental over the control treatment (or vice versa), since the trial is framed as a comparison between two different interventions previously described in the literature. It is worth noting that the distinction between motor execution and motor imagery is often imperceptible, even for professionals in the field, who have often described voluntary movements of the missing limb as imaginary movements. ${ }^{9}{ }^{32-37}$ We take this fact as a corroborant of our assumption that there are no differences at baseline with respect to expectations and opinions about the assigned treatment among participants. Nevertheless, individuals' expectations regarding outcomes and credibility of the assigned treatment are assessed with the EXPECT-SF and the OAT questionnaires, respectively.

The nature of the investigation does not allow the masking of the treatment for the therapists. However, it is still important to check for possible differences between the two groups concerning the therapists' supportive behaviour. For this reason, the HCCQ is included as a measure of the extent to which a healthcare provider (or the staff) interacts with their patient in a supportive manner.

The outcome assessments are conducted by independent persons who are blinded to the group allocation, making the trial double blind. In order to keep group allocation confidential, participants are requested prior to each assessment not to reveal allocation or therapy content to the evaluators.

The raw data resulting from the outcome assessment has the same structure for both interventions, making it impossible to tell the group assignment without being in possession of the documents containing links between participant's identity and their code number.

\section{METHODS: DATA COLLECTION, MANAGEMENT AND ANALYSIS Data collection and management}

The monitor of the study (EL) is in charge of overseeing the progress of the RCT and ensuring that it is conducted, recorded and reported in accordance with the protocol, Good Clinical Practice (GCP) and regulatory requirements.
The monitor supplies case report forms (CRFs), which are filled in by the evaluator at each site. The evaluator is responsible to document all data obtained during the study, which is identified by participant code number. This also applies to data for patients who, after having consented to participate, undergo the baseline examinations required for inclusion in the study, but who are not included. No items in the CRF are to be left unattended: if data are missing or are impossible to obtain, these should be documented as 'not available' (NA) and the reasons for missing data must be noted in the document.

All data are recorded and stored in digital form on encrypted electronic devices. Documents containing links between a participant's identity and their code number exist only in paper form and are kept in locked file cabinets with limited access at the investigation site where the participants have been treated. In accordance with the regulations issued by The Swedish Data Protection Authority, a personal register will be established.

The clinical investigators are responsible to probe, via discussion with the participant, for the occurrence of adverse events during each visit and record the information in the patient CRF. Adverse events must be described by duration (start and stop dates and times), severity, outcome, treatment and relation to study device, or if unrelated, the cause. The investigator must report any reportable event to the monitor in acceptable timely conditions, but not later than three working days after the occurrence of the event.

The sponsor must report to the Medical Products Agency (Läkemedelsverket) any serious adverse event, which indicates an imminent risk of death, serious injury or serious illness and that requires prompt remedial action for other patients, users or other persons immediately, but not later than two working days after becoming aware of a new reportable event or of new information in relation to an already reported event.

Once all the data are collected, checked and corrected, the database is closed, and analyses performed. All data transfer, processing and analyses are done using depersonalised data, and all the data sets are protected by password. In order to promote data quality, the evaluators are trained on all the data collection and management procedures and are provided with written instructions by the first (EL) and last (MO-C) authors.

To incentivise the completion of the follow-up, the patients are given the choice to participate in these assessments at the clinic or via a phone interview with the evaluators. When possible, follow-up assessments are also conducted with participants that had discontinued the treatment or withdrew from the study.

\section{Statistical methods}

The main analysis will be performed in terms of change from baseline to the measurement at treatment completion using the intention to treat (ITT) population, namely all the participants enrolled into the study considered according to their intial allocation. Complementary 
analyses will be performed on the per-protocol (PP) population with respect to the change from baseline to the follow-up assessments at 1,3 and 6 months after completion. These complementary analyses will include also the data coming from patients that, after appropriate washout period to exclude carry-over, have crossed over to the alternative treatment. Both the ITT population and the PP population will be specified in detail at the Clean file meeting before the database lock and before breaking the code. The PP population will be restricted to the participants who successfully complete all 15 treatment sessions.

Suitable graphical and numerical summaries will be provided for all the variables measured and for corresponding changes in scores.

For the main unadjusted comparison between two groups, Fisher's non-parametric permutation test will be used for continuous variables, Mantel-Haenszel $\chi^{2}$ test for ordered categorical variables, Fisher's exact test for dichotomous variables and Pearson's $\chi^{2}$ test for non-ordered categorical variables. CIs at $95 \%$ for the mean differences between two groups will be given when appropriate. If differences exist between the two randomised groups between baseline variables that could influence the outcome variables, a complementary adjusted analysis will be performed for these baseline variables.

For adjusted comparison between two groups, analysis of covariance will be used for continuous outcome variables not obviously non-normally distributed with intervention/control as independent variable and all confounders as covariates.

For analysis of change within groups, Wilcoxon signedrank test will be used for continuous variables and sign test for ordered categorical and dichotomous variables. A complementary mixed model analysis between the two treatments regarding the primary efficacy variable with centre as random effect will be used to correct for the centre-effect in the statistical models.

All correlations will be performed with Spearman's correlation coefficient. The distribution of continuous variables will be given as mean, SD, median, minimum and maximum, and distribution of categorical variables will be given as numbers and percentages. All statistical tests will be two sided and conducted at the 5\% significance level. The theory of sequential multiple test procedures will be applied for the primary analysis and for secondary analyses. If a test gives a significant result at the $5 \%$ significance level, the total test mass will be transferred to the following number in the test sequence until a non-significant result is achieved. All these significant tests will be considered confirmative. A Statistical Analysis Plan will be written with all detailed statistical analyses specified.

\section{Patient and public involvement}

The design of the study was informed by the experience with our previous clinical investigation, ${ }^{6}$ thanks to which patients' priorities, experience and preferences were identified and used for the development of the research question and outcome measures of the current RCT. The burden of the control intervention was assessed with a pilot study on volunteers with past experience with the experimental intervention.

\section{Ethics and dissemination}

Research ethics approval

There are no known risks associated with the experimental or control treatments, and clinically significant deterioration is rare. Possible individual benefits include reduced PLP, reduced disability associated with pain and improvement in various aspects related to quality of life. This trial has been approved by the governing ethical committees of each participating country. Important protocol modifications will be reported in a timely manner to all the relevant parties.

\section{Access to data}

The principal investigator, MO-C, has full access to all of the data in the study except the documents containing the link between patient's identity and their code number, which will be accessible only after the completion of the data analyses. MO-C takes responsibility for the integrity of the data and the accuracy of the data analysis.

\section{Dissemination policy}

Regardless of the significance, direction or magnitude of effect, the consortium will publish the findings of this study in scientific, peer-reviewed journals and conferences following the Consolidated Standards of Reporting Trials guidelines. All the clinical investigators will author the scientific article reporting the results of the trial. Results will be also disseminated to all the participants of the study with a report. No professional writers external to the study will be used aside from conventional English proof reading. Access to the detailed clinical investigation plan, participant-level dataset and statistical code will be granted based on reasonable requests after the publication of the study.

\section{Trial status}

This clinical trial is currently in the participant enrolment phase. Fourteen patients have been randomised and are under treatment at November 2017. It is anticipated that full analysis will be finalised in April 2020.

\section{Author affiliations}

${ }^{1}$ Biomechatronics and Neurorehabilitation Laboratory, Department of Electrical Engineering, Chalmers University of Technology, Goteborg, Sweden

${ }^{2}$ Department of Prosthetics and Orthotics, Faculty of Medicine and Health, Örebro University Hospital, Örebro, Sweden

${ }^{3}$ Faculty of Medicine and Health, University Health Care Research Centre, Örebro University, Örebro, Sweden

${ }^{4}$ University Rehabilitation Institute, Ljubljana, Slovenia

${ }^{5}$ Faculty of Medicine, University of Ljubljana, Ljubljana, Slovenia

${ }^{6}$ Department of Rehabilitation Medicine, University of Groningen, University Medical Centre Groningen, Haren, Groningen, The Netherlands

${ }^{7}$ School of Psychology \& Centre for Pain Research, National University of Ireland, Galway, Ireland

${ }^{8}$ Centre for Advanced Reconstruction of Extremities, Sahlgrenska University Hospital, Gothenburg, Sweden

${ }^{9}$ Gåskolan/Ortopedteknik, Sahlgrenska Universitetssjukhuset, Goteborg, Sweden 
${ }^{10}$ Rehabcenter Sfären, Bräcke Diakoni, Stockholm, Sweden

${ }^{11}$ Institute of Biomedical Engineering, University of New Brunswick, Fredericton, New Brunswick, Canada

${ }^{12}$ Fysische Geneeskunde en Revalidatie, University Hospital Gent, Gent, Belgium ${ }^{13}$ Integrum AB, Molndal, Sweden

Acknowledgements The authors would like to thank the patients that with their feedback helped to define the research questions, outcome measures and the patient advisers.

Contributors $\mathrm{M} 0-\mathrm{C}$ conceived the phantom motor execution treatment. M0-C and EL reviewed the literature and designed the study. All authors provided feedback on the design of the trial. BEM and MP assisted in the selection of psychological measure. LB-K and KK-0 coordinated the ethical applications. EL and MO-C drafted the manuscript. All authors revised the study protocol and approved the final manuscript. MO-C is the coordinating investigator of the study and endpoint adjudication evaluator. EL is the monitor of the trial, independent from the sponsor, and responsible for data management. Each site is constituted by at least a principal investigator, a therapist and a blinded evaluator. Investigational sites are independent from each other and from the sponsor.

Funding This study was funded by the Promobilia foundation (F16501), VINNOVA (Medtech4Health 2016-02290), EFIC Grünenthal Grant (358041552) and Integrum $A B$ (sponsor). The sponsor (Integrum $A B$ ) provided the devices and materials used in this study.

Disclaimer Neither the sponsor nor the funders (Promobilia, VINNOVA, EGG) had a role in the design of the present protocol.

Competing interests The sponsor of this study (Integrum AB) is a for-profit organisation that might commercialise the device used in this study (phantom motor execution and phantom motor imagery). $\mathrm{MO}-\mathrm{C}$ was partially funded by Integrum AB. The core technology used in this study has been made freely available as open source by MO-C (machine learning, virtual reality and electronics).

Patient consent Not required.

Ethics approval Regionala Etikprövningsnämnden i Göteborg.

Provenance and peer review Not commissioned; externally peer reviewed.

Open access This is an open access article distributed in accordance with the Creative Commons Attribution Non Commercial (CC BY-NC 4.0) license, which permits others to distribute, remix, adapt, build upon this work non-commercially, and license their derivative works on different terms, provided the original work is properly cited and the use is non-commercial. See: http://creativecommons.org/ licenses/by-nc/4.0/

(c) Article author(s) (or their employer(s) unless otherwise stated in the text of the article) 2018. All rights reserved. No commercial use is permitted unless otherwise expressly granted.

\section{REFERENCES}

1. Dijkstra PU, Geertzen JH, Stewart R, et al. Phantom pain and risk factors: a multivariate analysis. J Pain Symptom Manage 2002;24:578-85.

2. Clark RL, Bowling FL, Jepson F, et al. Phantom limb pain after amputation in diabetic patients does not differ from that after amputation in nondiabetic patients. Pain 2013;154:729-32.

3. Nikolajsen L, Jensen TS, pain PlimbBr J Anaesth 2001;87:107-16.

4. Batsford S, Ryan CG, Martin DJ. Non-pharmacological conservative therapy for phantom limb pain: A systematic review of randomized controlled trials. Physiother Theory Pract 2017;33:173-83.

5. Ortiz-Catalan M, Sander N, Kristoffersen MB, et al. Treatment of phantom limb pain (PLP) based on augmented reality and gaming controlled by myoelectric pattern recognition: a case study of a chronic PLP patient. Front Neurosci 2014;8.

6. Ortiz-Catalan M, Guðmundsdóttir RA, Kristoffersen MB, et al. Phantom motor execution facilitated by machine learning and augmented reality as treatment for Phantom Limb Pain. Lancet 2016;388:2885-94

7. Raffin E, Richard N, Giraux P, et al. Primary motor cortex changes after amputation correlate with phantom limb pain and the ability to move the phantom limb. Neuroimage 2016;130:134-44.

8. Lendaro E, Mastinu E, Håkansson B, et al. Real-time Classification of Non-Weight Bearing Lower-Limb Movements Using EMG to Facilitate Phantom Motor Execution: Engineering and Case Study Application on Phantom Limb Pain. Front Neurol 2017;8.
9. Maclver K, Lloyd DM, Kelly S, et al. Phantom limb pain, cortical reorganization and the therapeutic effect of mental imagery. Brain 2008;131(Pt 8):2181-91.

10. Chan BL, Witt R, Charrow AP, et al. Mirror therapy for phantom limb pain. N Engl J Med 2007;357:2206-7.

11. Moseley GL. Graded motor imagery for pathologic pain: a randomized controlled trial. Neurology 2006;67:2129-34.

12. Bowering KJ, O'Connell NE, Tabor A, et al. The effects of graded motor imagery and its components on chronic pain: a systematic review and meta-analysis. J Pain2013;14:3-13.

13. Ortiz-Catalan $M$, Brånemark R, Håkansson B. BioPatRec: A modular research platform for the control of artificial limbs based on pattern recognition algorithms. Source Code Biol Med 2013;8:11.

14. Ortiz-Catalan M, Hkansson B, Brnemark R. Real-Time and Simultaneous Control of Artificial Limbs Based on Pattern Recognition Algorithms. IEEE Transactions on Neural Systems and Rehabilitation Engineering 2014;22:756-64.

15. Simon AM, Hargrove LJ, Lock BA, et al. Target Achievement Control Test: evaluating real-time myoelectric pattern-recognition control of multifunctional upper-limb prostheses. J Rehabil Res Dev 2011;48:619-27.

16. Melzack R. The short-form McGill Pain Questionnaire. Pain 1987;30:191-7.

17. Pollard CA. Preliminary validity study of the pain disability index. Percept Mot Skills 1984;59:974.

18. Melzack R. The McGill Pain Questionnaire: major properties and scoring methods. Pain 1975;1:277-99.

19. Herdman M, Gudex C, Lloyd A, et al. Development and preliminary testing of the new five-level version of EQ-5D (EQ-5D-5L). Qual Life Res 2011;20:1727-36.

20. Nicholas MK. The pain self-efficacy questionnaire: Taking pain into account. Eur J Pain 2007;11:153-63.

21. Nicholas MK, McGuire BE, Asghari A, et al. A 2-item short form of the Pain Self-efficacy Questionnaire: development and psychometric evaluation of PSEQ-2. J Pain 2015;16:153-63.

22. McWilliams LA, Kowal J, Wilson KG. Development and evaluation of short forms of the Pain Catastrophizing Scale and the Pain Selfefficacy Questionnaire. Eur J Pain 2015;19:1342-9.

23. Sullivan MJL, Bishop SR, Pivik J. The Pain Catastrophizing Scale: Development and validation. Psychol Assess 1995;7:524-32.

24. Sullivan MJ, Thorn B, Haythornthwaite JA et al. Theoretical perspectives on the relation between catastrophizing and pain. Clin $J$ Pain2001;17:52-64.

25. Spitzer RL, Kroenke K, Williams JBW. Validation and Utility of a Selfreport Version of PRIME-MD. J Am Med Assoc 1999;282:1737-44.

26. Kroenke K, Spitzer RL, Williams JB. The Patient Health Questionnaire-2: validity of a two-item depression screener. Med Care 2003;41:1284-92.

27. Hurst $\mathrm{H}$, Bolton J. Assessing the clinical significance of change scores recorded on subjective outcome measures. J Manipulative Physiol Ther 2004;27:26-35

28. Jones SMW, Lange J, Turner J, et al. Development and Validation of the EXPECT Questionnaire: Assessing Patient Expectations of Outcomes of Complementary and Alternative Medicine Treatments for Chronic Pain. The Journal of Alternative and Complementary Medicine 2016;22-936-46.

29. Mooney TK, Beth M, Gibbons C, et al. Credibility and the Relation of Credibility to Therapy Outcome. 2015;24:565-77.

30. Williams GC, Freedman ZR, Deci EL. Supporting autonomy to motivate patients with diabetes for glucose control. Diabetes Care 1998;21:1644-51

31. Saghaei M, Saghaei S. Implementation of an open-source customizable minimization program for allocation of patients to parallel groups in clinical trials. J Biomed Sci Eng 2011;04:734-9.

32. Ersland L, Rosén G, Lundervold A. Smievoll a I, Tillung T, Sundberg $\mathrm{H}$, et al. Phantom limb imaginary fingertapping causes primary motor cortex activation: an fMRI study. Neuroreport 1996;8:207-10.

33. Hugdahl K, Rosén G, Ersland L, et al. Common pathways in mental imagery and pain perception: an fMRI study of a subject with an amputated arm. Scand J Psychol 2001;42:269-75.

34. Lotze M, Flor H, Grodd W, et al. Phantom movements and pain. An fMRI study in upper limb amputees. Brain 2001;124(Pt 11):2268-77.

35. Rosén G, Hugdahl K, Ersland L, et al. Different brain areas activated during imagery of painful and non-painful 'finger movements' in a subject with an amputated arm. Neurocase2001;7:255-60.

36. Roux FE, Ibarrola D, Lazorthes $Y$, et al. Virtual movements activate primary sensorimotor areas in amputees: report of three cases. Neurosurgery 2001;49:736-41.

37. Roux FE, Lotterie JA, Cassol E, et al. Cortical areas involved in virtua movement of phantom limbs: comparison with normal subjects. Neurosurgery 2003;53:1342-53. 